\title{
Risk Of Urticaria In Geriatric Stroke Patients Who Received Influenza Vaccination: A Retrospective Cohort Study
}

This article was published in the following Dove Press journal: Clinical Interventions in Aging

Fai Lam, (iD ${ }^{1-4}$ Chun-Chuan Shih, ${ }^{5}$ TaLiang Chen, ${ }^{4,6}$ Chao-Shun Lin, (DD ${ }^{2-4}$ Hsiao-Ju Huang, ${ }^{7}$ Chun-Chieh Yeh, ${ }^{8,9}$ Yu-Chen Huang, ${ }^{10,11}$ Hung-Yi Chiou, ${ }^{1} *$ Chien-Chang Liao (iD) $2,4,11,12, *$

'School of Public Health, College of Public Health, Taipei Medical University, Taipei, Taiwan; ${ }^{2}$ Department of Anesthesiology, Taipei Medical University Hospital, Taipei, Taiwan; ${ }^{3}$ Anesthesiology and Health Policy Research Center, Taipei Medical University Hospital, Taipei, Taiwan; ${ }^{4}$ Department of Anesthesiology, School of Medicine, College of Medicine, Taipei Medical University, Taipei, Taiwan; ${ }^{5} \mathrm{~S}$ chool of Chinese Medicine for Post-Baccalaureate, I-Shou University, Kaohsiung, Taiwan; ${ }^{6}$ Department of Anesthesiology, Wan Fang Hospital, Taipei Medical University, Taipei, Taiwan; ${ }^{7}$ Devision of Chinese Medicine, An Nan Hospital, China Medical University, Tainan, Taiwan;

${ }^{8}$ Department of Surgery, China Medical University Hospital, Taichung, Taiwan; ${ }^{9}$ Department of Surgery, University of Illinois, Chicago, IL, USA; ${ }^{10}$ Department of Dermatology, Wan Fang Hospital, Taipei Medical University, Taipei, Taiwan;

"'Research Center of Big Data and MetaAnalysis, Wan Fang Hospital, Taipei Medical University, Taipei, Taiwan; ${ }^{12}$ School of Chinese Medicine, College of Chinese Medicine, China Medical University, Taichung, Taiwan

*These authors contributed equally to this work

Correspondence: Chien-Chang Liao Department of Anesthesiology, Taipei Medical University Hospital, Taipei I I0, Taiwan

Tel +886-2-27372I 81 ext. 8310

Fax +886-2-27367344

Email jacky48863027@yahoo.com.tw
Objective: Urticaria is a mast cell-related disease caused severe itching and the lifetime prevalence of urticaria is about $20 \%$ in general population. Our purpose is to evaluate risk of urticaria in geriatric stroke patients received influenza vaccination (IV).

Methods: In a cohort of 192,728 patients with newly diagnosed stroke aged over 65 years obtained from 23 million people in Taiwan's National Health Insurance between 2000 and 2008, we identified 9890 stroke patients who received IV and 9890 propensity score-matched stroke patients who did not receive IV. Controlling for immortal time bias, both the IV and non-IV groups were followed for one year. Urticaria events were identified during the follow-up period. We calculated the adjusted rate ratios (RRs) and 95\% confidence intervals (CIs) of the one-year risk of urticaria associated with IV.

Results: During the follow-up period of one year, stroke patients with IV had a significantly higher risk of urticaria compared with non-IV stroke patients (RR 1.81, 95\% CI 1.47-2.23). An increased risk of urticaria in stroke patients with IV was noted in both sexes, patients 65-84 years of age, patients with comorbid medical conditions, and various time intervals of follow-up. Vaccinated stroke patients with hemorrhage (RR 4.00, 95\% CI 1.76-9.10) and those who received intensive care (RR 5.14, 95\% CI 2.32-11.4) had a very high risk of urticaria compared with those without IV.

Conclusion: Receiving IV may be associated with an increased risk of urticaria in stroke patients. We could not infer the causality from the current results because of this study's limitations. Future investigations are needed to evaluate the possible mechanism underlying the association between IV and urticaria.

Keywords: urticaria, stroke, influenza vaccination

\section{Introduction}

Urticaria is an allergic disease that causes severe itching and pruritus that may affect the quality of life. ${ }^{1}$ The lifetime prevalence of urticaria is approximately $20 \%$ of the general population across all ages and up to $40 \%$ in the elderly. ${ }^{2,3}$ Urticaria is caused by mast cells, and the symptoms are wheals or angioedema or both on the patient's body. Factors known to trigger urticaria include drugs (opiates, nonsteroidal anti-inflammatory drugs, muscle relaxants, vancomycin), food ingestion, insect stings, and latex. ${ }^{4}$ However, some patients have urticaria without exposure to the known risk factors mentioned above, and the full range of triggering factors is not fully understood.

Influenza is considered to be a leading threat to global health; influenza caused at least 54,481,000 episodes (including 8,172,000 severe episodes) and 9,459,000 
hospitalizations worldwide in $2017 .^{5}$ The most effective method for influenza infection prevention and control is to receive the influenza vaccination (IV) annually. ${ }^{6}$ Several studies have suggested that IV not only prevents influenza infection but also lowers the risk of major adverse cardiovascular events, ${ }^{7}$ pneumonia, ${ }^{8}$ and stroke. ${ }^{9}$ Receiving the IV is also associated with reduced poststroke complications and mortality. ${ }^{10}$

The safety of the IV is also considered an important issue. The most common side effect is injection site soreness or pain $(20-60 \%) .^{11,12}$ Systemic effects such as low-grade fever, myalgia, headache, and fatigue are less common (10-20\%). ${ }^{11}$ Erythema, induration, swelling, and pruritus are also noted as common local adverse effects. Serious adverse events are rare. Immediate immunoglobulin (Ig) E-mediated hypersensitivity reactions after the IV seldom occur. ${ }^{12}$ However, a review report suggested that IV might be associated with urticaria. ${ }^{13}$ There were three case reports including 44 cases investigated the influence of IV on vasculitis and skin reactions. ${ }^{14-16}$ Two observational studies including 937 patients and a safety surveillance including 4.1 million adults and children in France also reported similar side effects. ${ }^{17-19}$ To the best of our knowledge, there is no evidence that urticaria occurred within a longer observational period ( $>6$ weeks) after IV.

Elderly individuals and those with a history of chronic disease, including stroke, are populations susceptible to influenza, ${ }^{20,21}$ and more than half of stroke patients are elderly. ${ }^{22}$ Using the research database of the National Health Insurance in Taiwan, we conducted this populationbased retrospective cohort study to evaluate the risk of urticaria in geriatric stroke patients who received the IV.

\section{Methods}

\section{Source Of Data}

Taiwan's National Health Insurance program was implemented in March 1995 and now covers more than 99\% of Taiwan's 23 million residents. We conducted this study by using the reimbursement claims data from this insurance program, which records all beneficiaries' medical services, including inpatient and outpatient demographic characteristics, physicians' clinical diagnoses, treatment procedures, medication prescriptions, and the corresponding medical expenditures. Scientific articles based on this database have been accepted for publication in prominent journals, and detailed comments, assessments, and reviews have also been documented. ${ }^{10,23-25}$ This study was approved by the institutional review board of Taipei Medical University (TMU-JIRB-201910032) and E-DA Hospital (TMU-JIRB-201701050; EDA-JIRB-2017144).

\section{Study Design}

We examined the medical claims of the 404,914 Taiwan residents who first underwent inpatient care for stroke (defined as a physician's primary diagnosis of cerebrovascular disease and hospitalization for more than one day) in 2000-2008 and identified 192,728 people aged $\geq 65$ years. The inclusion criteria of stroke patients in this study were that people who had first-time stroke hospitalization with physician's primary diagnosis of stroke during the inpatient medical care. The exclusion criteria were that people who had previous inpatient or outpatient care for stroke or they had any previous stroke history before the index date. Of those, 88,990 had received the IV after discharge from the index stroke admission. Each patient who underwent inpatient care for stroke and received the IV was randomly matched to an unvaccinated patient who underwent inpatient care for stroke using a propensity score matched-pair procedure to adjust for age, sex, low-income status, calendar years, hospital status, coexisting medical conditions, stroke types, and stroke admission characteristics. After propensity score matching (case-control ratio, 1:1), there were 9890 patients who received the IV and 9890 who did not. Both IV and non-IV groups were followed from the index date to the end of one year (365 days with adjustment for immortal time bias) or until they were censored because of death. Urticaria events were considered the study outcome during the follow-up period. For the nonIV group, the starting point of follow-up was the first day after discharge from stroke admission. For the IV group, the starting point of follow-up was the date of receiving the IV after discharge from the stroke admission.

\section{Measures And Definitions}

In this study, people with low-income status were defined as people who had a certificate of low income. According to the regulations from the Bureau of National Health Insurance in Taiwan, people with low-income status are qualified to have the registration fee and medical copayment waived when obtaining medical care. A medical center was defined as those with a qualification certificate, which means the hospital has a high volume and more professional medical workers. The International Classification of Diseases, Ninth Revision, Clinical Modification (ICD-9-CM) was used to define the physicians' diagnoses. We considered incident 
urticaria (ICD-9-CM 708) after stroke as the study outcome in the present investigation. Based on our previous studies, ${ }^{10,23,24}$ coexisting medical conditions determined from medical claims included hypertension (ICD-9-CM 401-405), diabetes (ICD-9-CM 250), ischemic heart disease (ICD-9-CM 410-414), chronic obstructive pulmonary disease (ICD-9-CM 491,492, 496), mental disorders (ICD9-CM 290-319), influenza (ICD-9-CM 480-487), viral hepatitis (ICD-9-CM 070), urinary tract infection (ICD9-CM 599.0), asthma (ICD-9-CM 493), cancer (ICD-9-CM 140-208, 230-234), dementia (ICD-9-CM 290 and 331.0), hyperlipidemia (ICD-9-CM 272.0, 272.1, 272.2, 272.4), heart failure (ICD-9-CM 428), pneumonia (ICD-9-CM 480-486), allergic rhinitis (ICD-9-CM 477), epilepsy (ICD9-CM 345), and liver cirrhosis (ICD-9-CM 571.2, 571.5, 571.6). Renal dialysis was defined by administrative codes (D8 and D9).

\section{Statistical Analyses}

We used propensity score-matched pair analysis to determine the association between IV and urticaria. A nonparsimonious multivariable logistic regression model was used to estimate propensity scores for stroke patients receiving IV or not. Clinical significance guided the initial choice of covariates in this model to include age, sex, low income, medical center, types of stroke, length of hospital stay, intensive care, neurosurgery, hypertension, diabetes, ischemic heart disease, chronic obstructive pulmonary disease, mental disorders, influenza, viral hepatitis, urinary tract infection, asthma, cancer, dementia, hyperlipidemia, heart failure, pneumonia, allergic rhinitis, epilepsy, liver cirrhosis, renal dialysis, and calendar year. We matched IV recipients to nonrecipients using a greedy matching algorithm (without replacement) with a caliper width of 0.2 SDs of the log odds of the estimated propensity score. Categorical variables are summarized with frequencies (percentages) and were compared between vaccinated patients and non-vaccinated patients with chi-square tests. We used $t$-tests to compare continuous variables that are summarized as the means \pm standard deviations between IV and non-IV groups. The adjusted rate ratios (RRs) and 95\% confidence intervals (CIs) of urticaria associated with IV were calculated using multivariate Poisson regression analyses with a generalized estimating equation. Additional analyses stratified by age, sex, number of medical conditions, and stroke type were also performed to examine urticaria after stroke among IV recipients within these strata. All analyses were performed using Statistical Analysis Software version 9.1 (SAS Institute Inc., Cary, NC, USA), and significance was determined by a two-sided probability value of $<0.05$.

\section{Results}

The baseline characteristics of the IV recipients and nonrecipients among stroke patients are shown in Table 1. As a result of the propensity score matching procedure, there were no significant differences in age, sex, low income status, medical center, types of stroke, length of hospital stay, intensive care, neurosurgery, hypertension, diabetes, ischemic heart disease, chronic obstructive pulmonary disease, mental disorders, influenza, viral hepatitis, urinary tract infection, asthma, cancer, dementia, hyperlipidemia, heart failure, pneumonia, allergic rhinitis, epilepsy, liver cirrhosis, renal dialysis, and calendar year between stroke patients with and without IV.

During the one-year follow-up period (Table 2), stroke patients with IV had a higher risk of urticaria compared with those without IV (RR 1.81, 95\% CI 1.47-2.23). The stratification analysis shows that IV was associated with an increased risk of urticaria among the following strata: men (RR 1.95, 95\% CI 1.47-2.57), women (RR 1.64, 95\% CI 1.20-2.25), 65-69 years of age (RR 1.57, 95\% CI 1.03-2.39), 70-74 years of age (RR $1.83,95 \%$ CI 1.23-2.74), 75-79 years of age (RR 2.10, 95\% CI 1.37-3.23), 80-84 years of age (RR 1.80, 95\% CI 1.05-3.09), without low income (RR 1.80, 95\% CI 1.46-2.23), in a medical center (RR 1.86, 95\% CI 1.30-2.68), not in a medical center (RR 1.78, 95\% CI 1.38-2.29), did not receive intensive care ( $R R$ 1.63, 95\% CI 1.31-2.02), and received intensive care (RR 5.14, 95\% CI 2.32-11.4). The association between IV and the risk of urticaria was also significant in people with hemorrhagic stroke (RR 4.00, 95\% CI 1.76-9.10), occlusion and stenosis (RR 1.78, 95\% CI 1.39-2.28), 0 medical conditions (RR 2.29, 95\% CI 1.54-3.42), 1 medical condition (RR 1.61, 95\% CI 1.18-2.18), $\geq 2$ medical conditions (RR 1.71 , 95\% CI 1.14-2.59), and hospital stays of 6-10 days ( $R R$ 2.96, 95\% CI 1.94-4.53) and $\geq 16$ days (RR $2.38,95 \%$ CI 1.44-3.95).

During the one-month follow-up after stroke admission (Table 3), patients who received the IV had a higher risk of urticaria than patients who did not receive the IV (RR 2.42, 95\% CI 1.40-4.21). During the two (RR 2.13, 95\% CI 1.40-3.25), three (RR 2.52, 95\% CI 1.71-3.71), four (RR 2.21, 95\% CI 1.57-3.12), five (RR 2.37, 95\% CI 1.71-3.27), six (RR 2.50, 95\% CI 1.84-3.39), seven (RR 2.35, 95\% CI 1.78-3.11), eight (RR 2.39, 95\% CI 
Table I Characteristics Of Geriatric Stroke Patients With And Without Influenza Vaccination

\begin{tabular}{|c|c|c|c|c|c|}
\hline & \multicolumn{2}{|c|}{$\begin{array}{l}\text { No IV } \\
(N=9890)\end{array}$} & \multicolumn{2}{|c|}{ IV $(\mathrm{N}=9890)$} & \multirow[t]{2}{*}{$P$} \\
\hline & $\mathbf{n}$ & (\%) & $\mathbf{n}$ & (\%) & \\
\hline Sex & & & & & 1.0000 \\
\hline Female & 4221 & $(42.7)$ & 4221 & (42.7) & \\
\hline Male & 5669 & (57.3) & 5669 & $(57.3)$ & \\
\hline Age, years & & & & & 1.0000 \\
\hline 65-69 & 2200 & (22.2) & 2200 & (22.2) & \\
\hline $70-74$ & 2602 & (26.3) & 2602 & (26.3) & \\
\hline $75-79$ & 2426 & (24.5) & 2426 & (24.5) & \\
\hline $80-84$ & 1642 & (16.6) & 1642 & (16.6) & \\
\hline$\geq 85$ & 1020 & $(10.3)$ & 1020 & $(10.3)$ & \\
\hline Low income & 92 & $(0.9)$ & 92 & $(0.9)$ & 1.0000 \\
\hline Medical center & 3441 & $(34.8)$ & $344 I$ & (34.8) & 1.0000 \\
\hline Subtypes of stroke & & & & & 1.0000 \\
\hline Hemorrhage & 1156 & $(11.7)$ & 1156 & (II.7) & \\
\hline Occlusion and stenosis & 7046 & (71.2) & 7046 & (7I.2) & \\
\hline Transient ischemic attack & 890 & $(9.0)$ & 890 & $(9.0)$ & \\
\hline Late effects of stroke & 791 & $(8.0)$ & 791 & (8.0) & \\
\hline Others & 7 & $(0.1)$ & 7 & $(0.1)$ & \\
\hline Stay in intensive care unit & 1049 & $(10.6)$ & 1049 & $(10.6)$ & 1.0000 \\
\hline Neurosurgery & 291 & $(2.9)$ & 291 & $(2.9)$ & 1.0000 \\
\hline Coexisting medical condition & & & & & \\
\hline Hypertension & 4947 & $(50.0)$ & 4947 & $(50.0)$ & 1.0000 \\
\hline Diabetes & 1820 & $(18.4)$ & 1820 & $(18.4)$ & 1.0000 \\
\hline Ischemic heart disease & 609 & $(6.2)$ & 609 & $(6.2)$ & 1.0000 \\
\hline COPD & 595 & $(6.0)$ & 595 & $(6.0)$ & 1.0000 \\
\hline Mental disorder & 503 & $(5.1)$ & 503 & $(5.1)$ & 1.0000 \\
\hline Influenza & 291 & $(2.9)$ & 291 & $(2.9)$ & 1.0000 \\
\hline Viral hepatitis & 173 & $(1.8)$ & 173 & $(1.8)$ & 1.0000 \\
\hline Urinary tract infection & 167 & $(1.7)$ & 167 & $(1.7)$ & 1.0000 \\
\hline Asthma & 134 & $(1.4)$ & 134 & $(1.4)$ & 1.0000 \\
\hline Cancer & 134 & $(1.4)$ & 134 & $(1.4)$ & 1.0000 \\
\hline Dementia & 124 & $(1.3)$ & 124 & $(1.3)$ & 1.0000 \\
\hline Hyperlipidemia & 120 & $(1.2)$ & 120 & $(1.2)$ & 1.0000 \\
\hline Congestive heart failure & 45 & $(0.5)$ & 45 & $(0.5)$ & 1.0000 \\
\hline Pneumonia & 38 & $(0.4)$ & 38 & $(0.4)$ & 1.0000 \\
\hline Allergic rhinitis & 36 & $(0.4)$ & 36 & $(0.4)$ & 1.0000 \\
\hline Dialysis & 8 & $(0.1)$ & 8 & $(0.1)$ & 1.0000 \\
\hline Epilepsy & 3 & $(0.0)$ & 3 & $(0.0)$ & 1.0000 \\
\hline Liver cirrhosis & 3 & $(0.0)$ & 3 & $(0.0)$ & 1.0000 \\
\hline Length of stay, days & & & & & 1.0000 \\
\hline $\mathrm{I}-5$ & 3875 & $(39.2)$ & 3875 & $(39.2)$ & \\
\hline $6-10$ & 2966 & $(30.0)$ & 2966 & $(30.0)$ & \\
\hline $11-15$ & 880 & (8.9) & 880 & (8.9) & \\
\hline$\geq 16$ & 2169 & $(21.9)$ & 2169 & (21.9) & \\
\hline Mean \pm SD & 12.18 & 16.36 & 12.04 & 15.62 & 0.5577 \\
\hline
\end{tabular}

(Continued)
Table I (Continued).

\begin{tabular}{|c|l|l|l|l|l|}
\hline \multirow{2}{*}{} & \multicolumn{2}{|l|}{$\begin{array}{l}\text { No IV } \\
\text { (N=9890) }\end{array}$} & \multicolumn{2}{l|}{ IV (N=9890) } & P \\
\cline { 2 - 5 } & $\mathbf{n}$ & $\mathbf{( \% )}$ & $\mathbf{n}$ & $\mathbf{( \% )}$ & \\
\hline Calendar year & & & & & \multirow{2}{*}{1.0000} \\
2000 & 1215 & $(12.3)$ & 1215 & $(12.3)$ & \\
2001 & 991 & $(10.0)$ & 991 & $(10.0)$ & \\
2002 & 998 & $(10.1)$ & 998 & $(10.1)$ & \\
2003 & 893 & $(9.0)$ & 893 & $(9.0)$ & \\
2004 & 1024 & $(10.4)$ & 1024 & $(10.4)$ & \\
2005 & 980 & $(9.9)$ & 980 & $(9.9)$ & \\
2006 & 1017 & $(10.3)$ & 1017 & $(10.3)$ & \\
2007 & 1235 & $(12.5)$ & 1235 & $(12.5)$ & \\
2008 & 1537 & $(15.5)$ & 1537 & $(15.5)$ & \\
\hline
\end{tabular}

Abbreviation: COPD, chronic obstructive pulmonary disease.

1.83-3.13), nine (RR 2.14, 95\% CI 1.67-2.73), ten (RR 2.00, 95\% CI 1.59-2.52), eleven (RR 1.81, 95\% CI 1.46-2.24), and twelve (RR 1.81, 95\% CI 1.47-2.23) months of follow-up, IV was associated with an increased risk of urticaria in stroke patients.

In the sensitivity analysis (Table 4), we found that patients who received an IV had an increased one-year risk of urticaria even after excluding the urticaria events within the first week (RR 1.77, 95\% CI 1.44-2.19), one month (RR 1.71, 95\% CI 1.38-2.13), two months (RR 1.73, 95\% CI 1.38-2.18), and three months (RR 1.64, 95\% CI 1.29-2.08) of the one-year period of follow-up. In supplementary Table S1, the factors associated with an increased risk of urticaria in geriatric stroke patients included transient ischemic attack (RR 1.87, 95\% CI 1.11-3.17), mental disorders (RR 1.53, 95\% CI 1.00-2.34), and asthma (RR 2.10, 95\% CI 1.07-4.10).

\section{Discussion}

To the best of our knowledge, our study is the first to show that receiving the IV is associated with an increased risk of urticaria in stroke patients during a one-year period of follow-up, and this association was significant in various subgroups, including both sexes, all age groups, patients with comorbid medical conditions, and patients with different subtypes of stroke. Even in the sensitivity analysis, the oneyear risk of urticaria remained associated with IV when excluding urticaria cases in the initial follow-up period.

The results of our study were similar to those of a previous investigation showing that stroke patients had many comorbid medical conditions, such as hypertension, coronary heart disease, heart failure, atrial fibrillation, 
Table 2 Overall And Stratified Analysis For The Risk Of Urticaria Associated With Influenza Vaccination In Geriatric Stroke Patients ${ }^{\dagger}$

\begin{tabular}{|c|c|c|c|c|c|c|}
\hline & \multicolumn{2}{|c|}{ No IV ( $\mathrm{N}=9890)$} & \multicolumn{2}{|c|}{ IV (N=9890) } & \multirow[b]{2}{*}{$\mathbf{R R}$} & \multirow[t]{2}{*}{$(95 \% \mathrm{CI})^{*}$} \\
\hline & Events & Rate, \% & Events & Rate, \% & & \\
\hline All & 135 & $\mathrm{I} .4$ & 244 & 2.5 & 1.81 & $(1.47-2.23)$ \\
\hline \multicolumn{7}{|l|}{ Sex } \\
\hline Female & 61 & 1.5 & 100 & 2.4 & 1.64 & $(1.20-2.25)$ \\
\hline Male & 74 & 1.3 & 144 & 2.5 & 1.95 & $(1.47-2.57)$ \\
\hline \multicolumn{7}{|l|}{ Age, years } \\
\hline $65-69$ & 33 & 1.6 & 55 & 2.5 & 1.57 & $(1.03-2.39)$ \\
\hline $70-74$ & 36 & 1.4 & 66 & 2.5 & 1.83 & $(1.23-2.74)$ \\
\hline $75-79$ & 30 & 1.2 & 63 & 2.6 & 2.10 & $(1.37-3.23)$ \\
\hline $80-84$ & 20 & 1.2 & 36 & 2.2 & 1.80 & $(1.05-3.09)$ \\
\hline$\geq 85$ & 14 & 1.4 & 24 & 2.4 & 1.71 & $(0.89-3.29)$ \\
\hline \multicolumn{7}{|l|}{ Low income } \\
\hline No & 133 & 1.4 & 240 & 2.5 & 1.80 & $(1.46-2.23)$ \\
\hline Yes & 2 & 2.2 & 4 & 4.4 & 2.00 & $(0.38-10.7)$ \\
\hline \multicolumn{7}{|l|}{ Medical center } \\
\hline No & 91 & 1.4 & 162 & 2.5 & 1.78 & $(1.38-2.29)$ \\
\hline Yes & 44 & 1.3 & 82 & 2.4 & 1.86 & $(1.30-2.68)$ \\
\hline \multicolumn{7}{|l|}{ Subtypes of stroke } \\
\hline Hemorrhage & 7 & 0.6 & 28 & 2.4 & 4.00 & $(1.76-9.10)$ \\
\hline Occlusion and stenosis & 96 & $\mathrm{I} .4$ & I7I & 2.4 & 1.78 & $(1.39-2.28)$ \\
\hline Transient ischemic attack & 20 & 2.3 & 23 & 2.6 & 1.15 & $(0.64-2.07)$ \\
\hline Late effects of stroke & 12 & 1.5 & 22 & 2.8 & 1.83 & $(0.91-3.68)$ \\
\hline \multicolumn{7}{|l|}{ Stay in intensive care unit } \\
\hline No & 128 & 1.5 & 208 & 2.4 & 1.63 & $(1.31-2.02)$ \\
\hline Yes & 7 & 0.7 & 36 & 3.4 & 5.14 & $(2.32-11.4)$ \\
\hline \multicolumn{7}{|l|}{ Number of medical conditions } \\
\hline 0 & 34 & 1.1 & 78 & 2.5 & 2.29 & $(1.54-3.42)$ \\
\hline 1 & 66 & 1.5 & 106 & 2.5 & 1.61 & $(1.18-2.18)$ \\
\hline$\geq 2$ & 35 & 1.4 & 60 & 2.5 & 1.71 & $(1.14-2.59)$ \\
\hline \multicolumn{7}{|l|}{ Length of stay, days } \\
\hline $\mathrm{I}-5$ & 68 & 1.8 & 88 & 2.3 & 1.29 & $(0.95-1.77)$ \\
\hline $6-10$ & 28 & 0.9 & 83 & 2.8 & 2.96 & $(1.94-4.53)$ \\
\hline $11-15$ & 18 & 2.1 & 23 & 2.6 & 1.28 & $(0.70-2.35)$ \\
\hline$\geq 16$ & 21 & 1.0 & 50 & 2.3 & 2.38 & (1.44-3.95) \\
\hline \multicolumn{7}{|l|}{ Calendar year } \\
\hline 2000 & 13 & 1.1 & 36 & 3.0 & 2.77 & $(1.49-5.16)$ \\
\hline 2001 & 14 & I. 4 & 32 & 3.2 & 2.29 & $(1.23-4.24)$ \\
\hline 2002 & 16 & 1.6 & 14 & 1.4 & 0.88 & $(0.43-1.77)$ \\
\hline 2003 & 15 & 1.7 & 30 & 3.4 & 2.00 & $(1.09-3.68)$ \\
\hline 2004 & 19 & 1.9 & 34 & 3.3 & 1.79 & $(1.03-3.10)$ \\
\hline 2005 & 14 & 1.4 & 27 & 2.8 & 1.93 & $(1.03-3.60)$ \\
\hline 2006 & 21 & 2.1 & 23 & 2.3 & 1.10 & $(0.61-1.96)$ \\
\hline 2007 & 10 & 0.8 & 28 & 2.3 & 2.80 & $(1.37-5.73)$ \\
\hline 2008 & 13 & 0.9 & 20 & I.3 & 1.54 & $(0.77-3.07)$ \\
\hline
\end{tabular}

Notes: *Adjusted all covariates listed in Table I. ${ }^{\dagger}$ The RR of influenza vaccination associated urticaria was $1.90(95 \% \mathrm{Cl} 1.54-2.35)$ after adjusted for all covariates listed in Table I plus urbanization and cardiovascular medications (as shown in Table S2).

Abbreviations: $\mathrm{Cl}$, confidence interval; $\mathrm{RR}$, rate ratio; IV, influenza vaccination. 
Table 3 Risk Of Urticaria Associated With Influenza Vaccination In Stroke Patients During Time Period Of Follow-Up

\begin{tabular}{|c|c|c|c|c|c|c|}
\hline \multirow{2}{*}{$\begin{array}{l}\text { Time } \\
\text { Period Of } \\
\text { Follow-Up }\end{array}$} & \multicolumn{2}{|c|}{$\begin{array}{l}\text { No IV } \\
(N=9890)\end{array}$} & \multicolumn{2}{|c|}{$\begin{array}{l}\text { IV } \\
(N=9890)\end{array}$} & \multirow[t]{2}{*}{$\mathbf{R} \mathbf{R}$} & \multirow[t]{2}{*}{$(95 \% \mathrm{CI})^{*}$} \\
\hline & Events & $\%$ & Events & $\%$ & & \\
\hline I week & 6 & 0.06 & 14 & 0.14 & 2.44 & $(0.94-6.34)$ \\
\hline I month & 18 & 0.18 & 42 & 0.42 & 2.42 & $(1.40-4.21)$ \\
\hline 2 months & 32 & 0.32 & 66 & 0.67 & 2.13 & $(1.40-3.25)$ \\
\hline 3 months & 36 & 0.36 & 88 & 0.89 & 2.52 & $(|.7|-3.7 \mid)$ \\
\hline 4 months & 47 & 0.48 & 101 & 1.02 & 2.21 & $(1.57-3.12)$ \\
\hline 5 months & 52 & 0.53 & 120 & 1.21 & 2.37 & $(1.7 I-3.27)$ \\
\hline 6 months & 58 & 0.59 & 142 & 1.43 & 2.50 & (1.84-3.39) \\
\hline 7 months & 70 & 0.71 & 162 & 1.64 & 2.35 & $(1.78-3.11)$ \\
\hline 8 months & 75 & 0.76 & 177 & 1.79 & 2.39 & $(1.83-3.13)$ \\
\hline 9 months & 93 & 0.94 & 197 & 1.99 & 2.14 & $(1.67-2.73)$ \\
\hline 10 months & 108 & 1.09 & 215 & 2.17 & 2.00 & (1.59-2.52) \\
\hline II months & 126 & 1.27 & 227 & 2.30 & 1.81 & $(1.46-2.24)$ \\
\hline 12 months & 135 & 1.37 & 244 & 2.47 & 1.81 & (1.47-2.23) \\
\hline
\end{tabular}

Note: *Adjusted all covariates listed in Table I.

Abbreviations: $\mathrm{Cl}$, confidence interval; IV, influenza vaccination; $\mathrm{RR}$, rate ratio.

peripheral arterial disease, dyslipidemia, and diabetes mellitus. ${ }^{22}$ The medications to treat these diseases might be associated with urticaria (such as angiotensin-converting enzyme inhibitors, angiotensin receptor blockers, aspirin and nonsteroidal anti-inflammatory drugs). ${ }^{4}$ Thus, urticaria might be more common in stroke patients, and limited information is known about the annual incidence of urticaria in stroke patients. Asthma and urticaria are both mast cellmediated diseases. Although the pathophysiology is different in asthma and urticaria, they are similar in terms of the histamine and inflammatory mast cell mediators released (such as interleukin IL-4, IL-10, IL-33 and B cell-activating factor). ${ }^{26-28}$ Some studies have shown that asthma and urticaria are related and that asthma is common in patients with chronic spontaneous urticaria. ${ }^{29,30}$

According to previous studies, ${ }^{4,13}$ the IV seldomly causes extensive cutaneous or chronic adverse effects. However, the most frequently reported events are injection site transient reactions or nonspecific inflammation. ${ }^{11}$ These events are usually self-limiting, and no special treatment is needed. Vasculitis, arthralgia, digestive disorders, abdominal pain, and respiratory symptoms have been sporadically reported in previous studies. ${ }^{15,31}$ Most of the patients recovered, and no further treatment was needed. In this study, we found that stroke patients who did or did not receive the IV differed in the presence and duration of urticaria.

To the best of our knowledge, information about the association between IV and chronic urticaria ( $>6$ weeks) and the potential mechanism was unavailable. We proposed some possible reasons that may help explain the increased risk of urticaria in stroke patients who have received the IV. First, systemic adverse effects are related to immediate or delayed-type hypersensitivity reactions. IV contains deactivated and purified influenza virus, antigens, hemagglutinins and neuraminidases that might trigger urticaria. ${ }^{32,33}$ Second, the components of the IV might also trigger urticaria. For example, the IV is produced in chicken eggs and contains a small amount of egg protein. Urticaria might be induced by eggs and other components, such as formaldehyde (inactivate toxins), aluminum salts (adjuvants, boost immune response), thimerosal (preservatives to prevent contamination), gelatin (vaccine stabilizer), antibiotics (vaccine storage in gentamicin or neomycin), could also contribute to urticaria. $^{4,34}$

Third, stroke patients who received the IV annually had better cognitive health and a greater ability to perform physical activity when compared with non-IV stroke patients. Vaccinated patients were healthier and had a lower risk of stroke when compared with nonvaccinated patients. ${ }^{35}$ The vaccinated patients not only had a greater chance of being vaccinated but also had likely accessed medical care more often. ${ }^{36}$ Due to the increased number of visits to the clinic and increased healthcare resource utilization, the vaccinated

Table 4 The Sensitivity Analysis For The One-Year Risk Of Urticaria Associated With Influenza Vaccination In Geriatric Stroke Patients

\begin{tabular}{|l|l|l|l|l|l|l|}
\hline \multirow{2}{*}{ Exclusion Of Urticaria Cases } & \multicolumn{2}{l|}{ No IV (N=9890) } & \multicolumn{2}{l|}{ IV (N=9890) } & \multirow{2}{*}{ RR* } \\
\cline { 2 - 6 } & Events & $\%$ & Events & $\%$ & \\
\hline First week after IV & 132 & 1.3 & 234 & 2.3 & 1.77 & $(1.44-2.19)$ \\
First I month after IV & 126 & 1.3 & 215 & 2.2 & 1.71 & $(1.38-2.13)$ \\
First 2 months after IV & 115 & 1.2 & 199 & 2.0 & 1.73 & $(1.38-2.18)$ \\
First 3 months after IV & 108 & 1.1 & 176 & 1.8 & 1.64 & $(1.29-2.08)$ \\
\hline
\end{tabular}

Note: *Adjusted all covariates listed in Table I.

Abbreviations: $\mathrm{Cl}$, confidence interval; IV, influenza vaccination; $\mathrm{RR}$, rate ratio. 
patients had a higher probability of having skin lesions noted and urticaria diagnosed. The difference in detection bias with regard to urticaria between the IV and non-IV groups is produced by surveillance bias. ${ }^{37}$ However, we performed a propensity-score matching procedure to reduce this type of bias in this study.

Fourth, people who received an IV were more likely to have better knowledge of, attitude towards and practice of health promoting activities and self-care. ${ }^{38,39}$ Previous studies also showed that this population had a higher proportion of patients with better health awareness, who are more likely to change their lifestyle factors (such as physical activity, eating habits) and seek complementary and alternative medicine when they have an illness or disease. ${ }^{23,40,41}$ This additional therapy or treatment (such as herbal remedy use, acupuncture, dietary supplementation) might increase the risk of urticaria. In our study, the use of complementary and alternative medicine could not be matched or investigated.

There were some limitations in this study. First, this study was performed based on insurance data. Due to the nature of the database, we did not have detailed information on lifestyle factors (such as alcohol consumption, smoking habits, physical activity), living environment, physical checkup results, biochemical examination and imaging findings. The clinical risk score of stroke severity, such as the National Institutes of Health Stroke Scale or the Barthel Index, was not known. Unmeasured confounding effects might exist. Second, the use of complementary and alternative medicines, surveillance bias, and other possible residual confounding biases could not be excluded from this study. We used a propensity score to balance the baseline characteristics between stroke patients who did or did not receive the IV. Even so, residual confounding bias might interfere with this study's findings. Third, our study was based on reimbursement claims data from Taiwan's National Health Insurance Program. If the patient did not seek medical care for urticaria or other comorbid medical conditions, the diagnosis could not be made. Thus, the occurrence of the disease might be underestimated. However, the possible underestimation should be equally distributed between both groups, thus minimizing this bias. Fourth, our study is based on the data within the years between 2000 and 2009. It is a study limitation that we could not reach the latest data to perform the analysis. The fifth, Although the ICD-9 codes of stroke and physician's diagnosis were validated in some studies, ${ }^{42,43}$ the validation of ICD-9 codes of urticaria has not been evaluated previously. It is also one of limitations in this study. In addition, the manufacturing process for the IV and the components might not be the same in all the IVs. We could not investigate the variations in contents of the IVs because the IVs came from different manufacturers, including those in France, the United Kingdom, and Taiwan. Finally, a causal relationship between the IV and urticaria risk could not be established because our study was not a prospective cohort study or randomized clinical trial.

In conclusion, we discovered the possibility that urticaria might be a side effect of the IV and that the risk of urticaria may last at least one year. However, we could not infer the causality from the current results because of this study's limitations. The management of side effects of IV was suggested to be considered carefully in stroke patients. Future population-based prospective study (at least 5000 persons with IV and 5000 persons without IV) is needed to provide causality and mechanisms by which IV increases urticaria risk during follow-up of one year.

\section{Abbreviations}

$\mathrm{CI}$, confidence interval; IV, influenza vaccination; RR, rate ratio; ICD-9-CM, International Classification of Diseases, 9th Revision, Clinical Modification.

\section{Author Contributions}

All authors contributed to data analysis, drafting and revising the article, gave final approval of the version to be published, and agree to be accountable for all aspects of the work.

\section{Funding}

This study was supported in part by the Taiwan's Ministry of Science and Technology (MOST108-2320-B-214-003MY3; MOST108-2221-E-038-006; MOST107-2221-E-038009; MOST106-2314-B-038-036-MY3).

\section{Disclosure}

The authors report no conflicts of interest in this work.

\section{References}

1. Baiardini I, Giardini A, Pasquali M, et al. Quality of life and patients' satisfaction in chronic urticaria and respiratory allergy. Allergy. 2003;58(7):621-623. doi:10.1034/j.1398-9995.2003.00091.x

2. Maurer M, Weller K, Bindslev-Jensen C, et al. Unmet clinical needs in chronic spontaneous urticaria. A GA ${ }^{2} \mathrm{LEN}$ task force report. Allergy. 2011;66(3):317-330. doi:10.1111/all.2011.66.issue-3 
3. Weisshaar E, Dalgard F. Epidemiology of itch: adding to the burden of skin morbidity. Acta Derm Venereol. 2009;89(4):339-350. doi:10.2340/00015555-0662

4. Radonjic-Hoesli S, Hofmeier KS, Micaletto S, et al. Urticaria and angioedema: an update on classification and pathogenesis. Clin Rev Allergy Immunol. 2018;54(1):88-101. doi:10.1007/s12016-017-8628-1

5. GBD 2017 Influenza Collaborators. Mortality, morbidity, and hospitalisations due to influenza lower respiratory tract infections, 2017: an analysis for the global burden of disease study 2017. Lancet Respir Med. 2019;7(1):69-89. doi:10.1016/S2213-2600(18)30496-X

6. Paules C, Subbarao K. Influenza. Lancet. 2017;390(10095):697-708. doi:10.1016/S0140-6736(17)30129-0

7. Udell JA, Zawi R, Bhatt DL, et al. Association between influenza vaccination and cardiovascular outcomes in high-risk patients: a meta-analysis. JAMA. 2013;310(16):1711-1720. doi:10.1001/jama.2013.279206

8. Grijalva CG, Zhu Y, Williams DJ, et al. Association between hospitalization with community-acquired laboratory-confirmed influenza pneumonia and prior receipt of influenza vaccination. JAMA. 2015;314(14):1488-1497. doi:10.1001/jama.2015.12160

9. Lee KR, Bae JH, Hwang IC, et al. Effect of influenza vaccination on risk of stroke: a systematic review and meta-analysis. Neuroepidemiology. 2017;48(3-4):103-110. doi:10.1159/000478017

10. Lam F, Chen TL, Shih CC, et al. Protective effect of influenza vaccination on outcomes in geriatric stroke patients: a nationwide matched cohort study. Atherosclerosis. 2019;282:85-90. doi:10.1016/ j.atherosclerosis.2019.01.008

11. Meijer WJ, Wensing AMJ, Bos AA, et al. Influenza vaccination in healthcare workers; comparison of side effects and preferred route of administration of intradermal versus intramuscular administration. Vaccine. 2017;35(11):1517-1523. doi:10.1016/j.vaccine.2017.01.065

12. Nichol KL, Margolis KL, Lind A, et al. Side effects associated with influenza vaccination in healthy working adults. A randomized, placebo-controlled trial. Arch Intern Med. 1996;156(14):1546-1550. doi:10.1001/archinte.1996.00440130090009

13. Barbaud A, Deschildre A, Waton J, Raison-Peyron N, Tréchot P. Hypersensitivity and vaccines: an update. Eur J Dermatol. 2013;23 (2):135-141. doi:10.1684/ejd.2012.1842

14. Barbaud A, Reichert-Pénétrat S, Béné MC, et al. Mécanisme immuno-pathologiques en cause dans un cas pédiatrique d'urticaire survenue lors de la primo-vaccination par vaccin anti-hépatite B. Ann Dermatol Venereol. 2000;127(6-7):662-663.

15. Watanabe T. Henoch-Schönlein purpura following influenza vaccinations during the pandemic of influenza A (H1N1). Pediatr Nephrol. 2011;26(5):795-798. doi:10.1007/s00467-010-1722-8

16. Barbaud A, Schmutz JL, Mougeolle JM. Réactions immunoallergiques cutanées dues aux vaccins. Ann Dermatol Venereol. 1995;122 (2):129-138.

17. Siret-Alatrista A, Bouali F, Demoly M, Bousquet PJ, Demoly P. The 2009-2010 H1N1 vaccination campaign for patients with egg allergy in a region of France. Allergy. 2011;66(2):298-299. doi:10.1111/all.2010.66. issue-2

18. Gagnon R, Primeau MN, Des Roches A, et al. Safe vaccination of patients with egg allergy with an adjuvanted pandemic H1N1 vaccine. J Allergy Clin Immunol. 2010;126(2):317-323. doi:10.1016/j.jaci.2010.05.037

19. Caillet C, Durrieu G, Jacquet A, et al. Safety surveillance of influenza A(H1N1)v monovalent vaccines during the 2009-2010 mass vaccination campaign in France. Eur J Clin Pharmacol. 2011;67 (6):649-651. doi:10.1007/s00228-010-0961-8

20. Thompson WW, Shay DK, Weintraub E, et al. Mortality associated with influenza and respiratory syncytial virus in the United States. JAMA. 2003;289(2):179-186. doi:10.1001/jama.289.2.179

21. Preaud E, Durand L, Macabeo B, et al. Annual public health and economic benefits of seasonal influenza vaccination: a European estimate. BMC Public Health. 2014;14:813. doi:10.1186/1471-2458-14-813
22. Roger VL, Go AS, Lloyd-Jones DM, et al. Heart disease and stroke statistics-2011 update: a report from the American Heart Association. Circulation. 2011;123(4):e18-e209.

23. Weng SW, Chen TL, Yeh CC, et al. An investigation of the use of acupuncture in stroke patients in Taiwan: a national cohort study. BMC Complement Altern Med. 2016;16(1):321. doi:10.1186/s12906016-1272-0

24. Liu WC, Lin CS, Yeh CC, et al. Effect of influenza vaccination against postoperative pneumonia and mortality for geriatric patients receiving major surgery: a nationwide matched study. $J$ Infect Dis. 2018;217(5):816-826. doi:10.1093/infdis/jix616

25. Hsieh CY, Su CC, Shao SC, et al. Taiwan's national health insurance research database: past and future. Clin Epidemiol. 2019;11:349-358. doi:10.2147/CLEP.S196293

26. Papadopoulos J, Karpouzis A, Tentes J, Kouskoukis C. Assessment of interleukins IL-4, IL-6. IL-8, IL-10 in acute urticaria. J Clin Med Res. 2014;6(2):133-137. doi:10.14740/jocmr1645w

27. Saluja R, Khan M, Church M, Maurer M. The role of IL-33 and mast cells in allergy and inflammation. Clin Transl Allergy. 2015;5:33. doi:10.1186/s13601-015-0076-5

28. Kessel A, Yaacoby-Bianu K, Vadasz Z, et al. Elevated serum B-cell activating factor in patients with chronic urticaria. Hum Immunol. 2012;73(6):620-622. doi:10.1016/j.humimm.2012.03.016

29. Isik AR, Karakaya G, Celikel S, Demir AU, Kalyoncu AF. Association between asthma, rhinitis, and NSAID hypersensitivity in chronic urticaria patients and prevalence rates. Int Arch Allergy Immunol. 2009;150(3):299-306. doi:10.1159/000222683

30. Vadasz Z, Kessel A, Hershko AY, Maurer M, Toubi E. Seasonal exacerbation of asthma is frequently associated with recurrent episodes of acute urticaria. Int Arch Allergy Immunol. 2016;169 (4):263-266. doi:10.1159/000446183

31. Gagnon R, Primeau MN, Des Roches A, et al. PHAC-CIHR Influenza Research Network. Safe vaccination of patients with egg allergy with an adjuvanted pandemic H1N1 vaccine. J Allergy Clin Immunol. 2010;126(2):317-323. doi:10.1016/j.jaci.2010.05.037

32. McMahon AW, Iskander JK, Haber P, Braun MM, Ball R. Inactivated influenza vaccine (IIV) in children $<2$ years of age: examination of selected adverse events reported to the Vaccine Adverse Event Reporting System (VAERS) after thimerosal-free or thimerosalcontaining vaccine. Vaccine. 2008;26(3):427-429. doi:10.1016/j. vaccine.2007.10.071

33. Wedi B, Raap U, Wieczorek D, Kapp A 1. Urticaria and infections. Allergy Asthma Clin Immunol. 2009;5(1):10. doi:10.1186/1710-1492$5-10$

34. Ponvert C, Scheinmann P. Vaccine allergy and pseudo-allergy. Eur J Dermatol. 2003;13(1):10-15.

35. Jackson LA, Jackson ML, Nelson JC, Neuzil KM, Weiss NS. Evidence of bias in estimates of influenza vaccine effectiveness in seniors. Int $J$ Epidemiol. 2006;35(2):337-344. doi:10.1093/ije/ dyi274

36. Lee IC, Chang CS, Du PL. Do healthier lifestyles lead to less utilization of healthcare resources? BMC Health Serv Res. 2017;17 (1):243. doi:10.1186/s12913-017-2185-4

37. Jackson ML, Yu O, Nelson JC, et al. Further evidence for bias in observational studies of influenza vaccine effectiveness: the 2009 influenza A(H1N1) pandemic. Am $J$ Epidemiol. 2013;178 (8):1327-1336. doi:10.1093/aje/kwt124

38. Lu PJ, O'Halloran A, Kennedy ED, et al. Awareness among adults of vaccine-preventable diseases and recommended vaccinations, United States, 2015. Vaccine. 2017;35(23):3104-3115. doi:10.1016/j.vaccine. 2017.04.028

39. Williams WW, Lu PJ, O'Halloran A, et al. Surveillance of vaccination coverage among adult populations - United States, 2015. MMWR Surveill Summ. 2017;66(11):1-28. doi:10.15585/mmwr.ss6611a1 
40. Templeton AJ, Thürlimann B, Baumann M, et al. Cross-sectional study of self-reported physical activity, eating habits and use of complementary medicine in breast cancer survivors. BMC Cancer. 2013;13:153. doi:10.1186/1471-2407-13-153

41. Fabian E, Töscher S, Elmadfa I, Pieber TR. Use of complementary and alternative medicine supplements in patients with diabetes mellitus. Ann Nutr Metab. 2011;58(2):101-108. doi:10.1159/ 000326765
42. Cheng CL, Chien HC, Lee CH, Lin SJ, Yang YH. Validity of in-hospital mortality data among patients with acute myocardial infarction or stroke in national health insurance research database in Taiwan. Int J Cardiol. 2015;201:96-101. doi:10.1016/j.ijcard.2015.07.075

43. Cheng CL, Kao YH, Lin SJ, Lee CH, Lai ML. Validation of the national health insurance research database with ischemic stroke cases in Taiwan. Pharmacoepidemiol Drug Saf. 2011;20 (3):236-242. doi:10.1002/pds.2087
Clinical Interventions in Aging

\section{Publish your work in this journal}

Clinical Interventions in Aging is an international, peer-reviewed journal focusing on evidence-based reports on the value or lack thereof of treatments intended to prevent or delay the onset of maladaptive correlates of aging in human beings. This journal is indexed on PubMed Central, MedLine, CAS, Scopus and the Elsevie
Dovepress

Bibliographic databases. The manuscript management system is completely online and includes a very quick and fair peer-review system, which is all easy to use. Visit http://www.dovepress.com/ testimonials.php to read real quotes from published authors. 